\title{
How equilibrium radionuclide angiography can quantify tricuspid regurgitation
}

\author{
Dominik C. Benz, MD, ${ }^{a}$ and Tobias A. Fuchs, $M^{a}$ \\ a Cardiac Imaging, Department of Nuclear Medicine, University Hospital Zurich, Zurich, \\ Switzerland
}

Received Jul 11, 2019; accepted Jul 11, 2019

doi: $10.1007 / \mathrm{s} 12350-019-01826-5$

\section{See related article, pp. 864-872}

In the United States, about 1.6 million patients suffer from moderate to severe tricuspid regurgitation (TR). ${ }^{1}$ In the majority of these cases $(>90 \%)$, regurgitation is functional or, in other words, secondary to the negative remodeling of the right ventricle (RV) and the tricuspid annulus. ${ }^{2}$ This remodeling may result from left-sided heart disease (e.g., aortic or mitral valve disease or left ventricular dysfunction) or pulmonary hypertension. In the remaining patients, the problem is the tricuspid valve itself. Primary (organic) tricuspid regurgitation can be observed in patients with congenital heart disease (e.g., Ebstein anomaly) or endocarditis, after trauma (e.g., endomyocardial biopsy), or induced by an intracardiac device lead. Treatment of the primary disease in patients with secondary tricuspid regurgitation as well as sufficient diuretic therapy is crucial. Despite the high prevalence, less than 8000 patients per year undergo tricuspid valve surgery. ${ }^{1}$ Its surgical management precipitates significant perioperative morbidity and mortality due to RV dysfunction and pulmonary hypertension. ${ }^{3}$ Additionally, timing of surgical intervention remains controversial for different reasons and due to limited data.

Regarding primary tricuspid regurgitation, it is indicated to treat severe regurgitation surgically in symptomatic patients without severe RV dysfunction, and to consider surgical treatment in asymptomatic patients with progressive $\mathrm{RV}$ dilatation or decline of $\mathrm{RV}$

Reprint requests: Tobias A. Fuchs, MD, Cardiac Imaging, Department of Nuclear Medicine, University Hospital Zurich, Ramistrasse 100, 8091 Zurich, Switzerland; tobias.fuchs@usz.ch

J Nucl Cardiol 2021;28:873-5.

$1071-3581 / \$ 34.00$

Copyright (C) 2019 American Society of Nuclear Cardiology. function. ${ }^{4}$ In patients with severe functional tricuspid regurgitation, surgical management of the tricuspid valve is indicated at the time of left-sided valve surgery. If functional tricuspid regurgitation is mild to moderate, surgery should be considered in patients with a dilated annulus and may be considered in patients with a previously documented right heart failure. ${ }^{4}$ Finally, there is a small but peculiar subgroup of patients with severe functional tricuspid regurgitation after left-sided surgery and without recurrent left-sided valve dysfunction (about 13\% of overall isolated tricuspid regurgitation surgery in the United States). ${ }^{5}$ Those patients who often experience severe symptoms are challenging: conservative treatment options are limited and redo surgery carries a high risk. Since RV dysfunction but not tricuspid regurgitation itself is independently associated with worse outcome in this setting, ${ }^{6}$ surgery should only be considered if RV dysfunction/dilatation is progressive but not yet severe and in the absence of severe left ventricular (LV) dysfunction and severe pulmonary hypertension. ${ }^{4}$ Despite all these elaborated recommendations, perioperative mortality remains strikingly high, ranging between $4 \%$ and $17 \% .^{7}$ Consequently, novel, less-invasive transcatheter tricuspid valve interventions gained interest over the past decade, ${ }^{8}$ and the initial global experience with these interventions has been encouraging. ${ }^{9}$ Comprehensive and accurate assessment of the tricuspid valve and RV, nonetheless, is mandatory in order to guide optimal therapy in those patients.

Echocardiographic examination is the primary tool to categorize tricuspid regurgitation into mild, moderate, and severe. ${ }^{10}$ However, grading the severity of tricuspid regurgitation by echocardiography is challenging, whether by qualitative, semiquantitative, or quantitative techniques due to the complex anatomy of the valve. ${ }^{11}$ Three-dimensional transthoracic and transoesophageal echocardiography overcome some limitations allowing more reliable and more accurate measurements. As stated above, assessment of RV function is essential due 
to the impact on treatment strategy. Nonetheless, although the assessment of RV function-of utmost importance for risk stratification and patient management-is feasible by echocardiography, cardiac magnetic resonance (CMR) provides an accurate and highly reproducible assessment of $\mathrm{RV}$ volumes and function. There are three methods to quantify tricuspid regurgitation from CMR. The most established (and most accurate) method to quantify the regurgitate volume of the tricuspid valve by CMR is subtracting pulmonary forward volume (measured in phase contrast imaging) from the RV stroke volume. Alternatively-in the absence of aortic regurgitation-aortic forward volume can be subtracted from the RV stroke volume. Lastly, in the absence of other regurgitate lesions or intracardiac shunts, LV stroke volume can be subtracted from RV stroke volume to obtain tricuspid regurgitation volume. Nevertheless, not all patients are suitable for a CMR examination, such as patients with non-magnetic resonance imaging conditional devices or claustrophobia.

Equilibrium radionuclide ventriculography (ERV) can be used to quantify LV and RV stroke volumes and ejection fractions. Similarly to CMR, LV and RV stroke volumes can be subtracted from each other and regurgitate volume can be quantified. However, the subtraction of stroke volumes can only be used in patients with single valve disease. As a result, this method (including CMR and ERV) is not feasible in patients with secondary tricuspid regurgitation due to mitral valve regurgitation. Both, planar and tomographic ECG-gated ERV can be acquired with technetium-99mpertechnetat-labeled red blood cells. As the amount of radioactivity in each chamber is proportional to the blood volume contained, quantitative indices (including $\mathrm{LV}$ ejection fraction and stroke volume) of the chambers can be assessed. In the left anterior oblique view (where the LV is best separated from the RV), LV ejection fraction can be calculated accurately in planar equilibrium radionuclide ventriculography. Due to the overlapping chambers, however, RV function cannot be easily estimated. By contrast, tomographic equilibrium radionuclide ventriculography (t-ERV) allows for better separation of the cardiac cavities and can overcome this issue without any geometrical assumptions. This is particularly important for the evaluation of RV function and RV stroke volume and has previously been validated against CMR with excellent reproducibility. ${ }^{12}$ Thanks to $\gamma$-cameras equipped with cadmium-zinc-telluride crystals, acquisition time has been shortened and radiation dose has been reduced. It is recommended to use at least 16 frames per cycle in order to avoid underestimation of ventricular volumes. Images are usually reconstructed and processed with commercially available semi-automated or fully automated software packages.

In the current issue of the Journal of Nuclear Cardiology, Eyharts et al. investigated the accuracy tERV for the quantification of tricuspid regurgitation by subtraction of LV and RV stroke volumes. ${ }^{13}$ The authors retrospectively included 61 patients who underwent both t-ERV and transthoracic echocardiography of whom a subgroup of 22 patients had an additional CMR. The tricuspid regurgitate volume was calculated by proximal isovelocity surface area (PISA) from TTE and by the volumetric method from t-ERV and CMR. The authors found an overestimation of tricuspid regurgitate volume by t-ERV compared with TTE (with a mean difference of $8 \mathrm{~mL})$ but a good correlation $(R=0.89 ; P<.0001)$. Among the subgroup of 22 patients who additionally underwent CMR, t-ERV again showed an overestimation of t-ERV against CMR (with a mean difference of $24 \mathrm{~mL}$ ). Interestingly, as illustrated in the correlation graphs, RV volumes were higher with t-ERV compared to CMR, and LV volumes were lower. The resulting higher RV stroke volume and lower LV stroke volume by t-ERV might explain the higher stroke volume difference leading to increased regurgitate volume for $\mathrm{t}$ ERV. Based on these results, the authors concluded that t-ERV for tricuspid regurgitation is not inferior to CMR considering TTE flow convergence method as the gold standard. Tricuspid regurgitation severity grading by $\mathrm{t}-$ ERV was particularly consistent with TTE for patients with severe tricuspid regurgitation (94\%).

Different methods do not certainly measure the same. Especially, the PISA method estimates a regurgitate volume whereas t-ERV and CMR quantify true volumes. The PISA methods suffers from pitfalls such as asymmetric dilatation of the tricuspid annulus, ellipsoidal orifice of the tricuspid regurgitation-jet, and angulation errors. A previous study showed only moderate agreement between echocardiography and the conventional CMR methodology for assessment of mitral regurgitation. ${ }^{14}$ Additionally, reference values differ between echocardiography and ERV. ${ }^{15,16}$ Moreover, while LV volume and LV ejection fraction by t-ERV are known to be highly reproducible, they may have significant variations depending on the software package. ${ }^{17,18}$

In summary, Eyharts et al. certainly stimulate the interest in t-ERV to gain more information than simply ventricular volumes and ejection fraction. However, the stroke volume method for quantification of tricuspid regurgitation is limited to patients without additional valvular diseases or shunts. Furthermore, while accurate quantification of the severity of tricuspid regurgitation is an essential step to guide further therapy, the treatment 
of tricuspid valve regurgitation is complex, integrating additional multi-parametric information such as mechanism, annular size, and RV volumes and function.

\section{Disclosures}

The University Hospital Zurich holds a research agreement with GE Healthcare. Dr. Benz reports a research grant from the Theodor and Ida Herzog-Egli-Foundation. Dr. Fuchs declares that he has no conflict of interest.

\section{References}

1. Stuge O, Liddicoat J. Emerging opportunities for cardiac surgeons within structural heart disease. J Thorac Cardiovasc Surg 2006;132:1258-61.

2. Rodés-Cabau J, Taramasso M, O'Gara PT. Diagnosis and treatment of tricuspid valve disease: Current and future perspectives. Lancet 2016;388:2431-42.

3. Kim YJ, Kwon DA, Kim HK, Park JS, Hahn S, Kim KH, et al. Determinants of surgical outcome in patients with isolated tricuspid regurgitation. Circulation 2009;120:1672-8.

4. Baumgartner H, Falk V, Bax JJ, De Bonis M, Hamm C, Holm PJ, et al. 2017 ESC/EACTS guidelines for the management of valvular heart disease. Eur Heart J 2017;38:2739-91.

5. Zack CJ, Fender EA, Chandrashekar P, Reddy YNV, Bennett CE, Stulak JM, et al. National trends and outcomes in isolated tricuspid valve surgery. J Am Coll Cardiol 2017;70:2953-60.

6. Kammerlander AA, Marzluf BA, Graf A, Bachmann A, Kocher A, Bonderman D, et al. Right ventricular dysfunction, but not tricuspid regurgitation, is associated with outcome late after left heart valve procedure. J Am Coll Cardiol 2014;64:2633-42.

7. Asmarats L, Taramasso M, Rodés-Cabau J. Tricuspid valve disease: Diagnosis, prognosis and management of a rapidly evolving field. Nat Rev Cardiol 2019. https://doi.org/10.1038/s41569-0190186-1.

8. Asmarats L, Puri R, Latib A, Navia JL, Rodés-Cabau J. Transcatheter tricuspid valve interventions: Landscape, challenges, and future directions. J Am Coll Cardiol 2018;71:2935-56.
9. Taramasso M, Alessandrini H, Latib A, Asami M, Attinger-Toller A, Biasco L, et al. Outcomes after current transcatheter tricuspid valve intervention: Mid-term results from the international TriValve registry. JACC Cardiovasc Interv 2019;12:155-65.

10. Grant AD, Thavendiranathan P, Rodriguez LL, Kwon D, Marwick TH. Development of a consensus algorithm to improve interobserver agreement and accuracy in the determination of tricuspid regurgitation severity. J Am Soc Echocardiogr 2014;27:277-84.

11. Dreyfus GD, Martin RP, Chan KM, Dulguerov F, Alexandrescu C. Functional tricuspid regurgitation: A need to revise our understanding. J Am Coll Cardiol 2015;65:2331-6.

12. Nichols K, Saouaf R, Ababneh AA, Barst RJ, Rosenbaum MS, Groch MW, et al. Validation of SPECT equilibrium radionuclide angiographic right ventricular parameters by cardiac magnetic resonance imaging. J Nucl Cardiol 2002;9:153-60.

13. Eyharts D, Lavie-Badie Y, Cazalbou S, Fournier P, Cariou E, Pascal P, et al. Quantitative assessment of tricuspid regurgitation using right and left ventricular stroke volumes obtained from tomographic equilibrium radionuclide ventriculography. J Nucl Cardiol 2019. https://doi.org/10.1007/s12350-019-01781-1.

14. Uretsky S, Gillam L, Lang R, Chaudhry FA, Argulian E, Supariwala A, et al. Discordance between echocardiography and MRI in the assessment of mitral regurgitation severity: A prospective multicenter trial. J Am Coll Cardiol 2015;65:1078-88.

15. Bellenger NG, Burgess MI, Ray SG, Lahiri A, Coats AJ, Cleland JG, et al. Comparison of left ventricular ejection fraction and volumes in heart failure by echocardiography, radionuclide ventriculography and cardiovascular magnetic resonance: Are they interchangeable? Eur Heart J 2000;21:1387-96.

16. Schwartz RG, Jain D, Storozynsky E. Traditional and novel methods to assess and prevent chemotherapy-related cardiac dysfunction noninvasively. J Nucl Cardiol 2013;20:443-64.

17. Chin BB, Bloomgarden DC, Xia W, Kim HJ, Fayad ZA, Ferrari VA, et al. Right and left ventricular volume and ejection fraction by tomographic gated blood-pool scintigraphy. J Nucl Med 1997;38:942-8.

18. Akinboboye O, Nichols K, Wang Y, Dim UR, Reichek N. Accuracy of radionuclide ventriculography assessed by magnetic resonance imaging in patients with abnormal left ventricles. J Nucl Cardiol 2005;12:418-27.

Publisher's Note Springer Nature remains neutral with regard to jurisdictional claims in published maps and institutional affiliations. 